\title{
Acute effects of breads prepared with $\beta$-glucan and black tea on glucose and insulin responses in healthy volunteers
}

\author{
A.M.M. Jalil, E. Combet, C.A. Edwards and A.L. Garcia \\ Human Nutrition, School of Medicine, College of Medical, Veterinary \& Life Sciences, University of Glasgow, New \\ Lister Building, Glasgow Royal Infirmary, 10-16 Alexandra Parade, Glasgow, G31 2ER, Scotland, United Kingdom
}

Obesity is a global problem affecting more than 1.9 billion adults in 2014 and is a major risk factor for developing chronic diseases ${ }^{(1)}$. Increasing dietary fibre intake is a key recommendation for weight control and prevention of chronic disease. In the UK, a minimum of $30 \mathrm{~g}$ of dietary fibre a day is recommended for the adult population ${ }^{(2)}$. However, dietary fibre intake in Western countries is generally less than adequate for the prevention of major non-communicable diseases (NCD) ${ }^{(3)}$. Thus finding alternative ways to increase dietary fibre consumption may help consumers achieve this recommendation. Fruits and vegetables are rich in (poly)phenols along with tea, coffee and cocoa, with tea one of the most important source of (poly)phenols in the UK diet ${ }^{(4)}$. High (poly)phenol intake is associated with lower risk of mortality ${ }^{(5)}$. Tea catechins positively regulated glucose levels in in vitro and in vivo studies. Combining the two functional ingredients ( $\beta$-glucan and black tea) in a staple food such as bread is a feasible way to simultaneously increase dietary fibre intake and deliver the health benefits of (poly)phenols. We have shown that combination of $\beta$-glucan and black tea in bread was acceptable, palatable and reduced the rate of in vitro starch hydrolysis ${ }^{(6)}$. Hence, we hypothesized that this bread would slow postprandial glucose and insulin responses in healthy volunteers.

Healthy subjects (7 males and 8 females; 25.5 (4.3) years; BMI $22.9(3.3) \mathrm{kg} / \mathrm{m}^{2}$ ) completed all four-arms of a cross-over trial. After an overnight fast, subjects were randomised to consume white bread (WB), or bread containing black tea (BT), $\beta$-glucan ( $\beta \mathrm{G})$ and both $\beta$-glucan and black tea ( $\beta$ GBT) breads for breakfast. All subjects consumed all breads with a one-week wash out between each leg of the trial. Blood samples were taken before $(0 \mathrm{~min})$ and at 15, 30, 60, 120 and $180 \mathrm{~min}$ after breakfast and assayed for glucose (ROCHE glucose diagnostic test, mmol/L, CV $<20 \%$ ) and insulin (Insulin ELISA kit, mU/L, CV $<50 \%$ ). Data was analysed using oneway ANOVA (between bread difference) and two-way ANOVA (time interactions difference) and $\mathrm{p}<0.05$ is considered as significant.

We observed high inter-individual variations in both glucose and insulin responses. There was a significant $(\mathrm{p}<0.05)$ difference in glucose response between $\beta G$ and $\beta G B T$ at 15 min (Fig. 1). There were significant $(p<0 \cdot 05)$ time interactions (fasting vs 30 min, 30 min vs $180 \mathrm{~min}$ ) for $\mathrm{WB}, \mathrm{BT}$ and $\beta \mathrm{GBT}$ except $\beta \mathrm{G}$. $\beta \mathrm{G}$ showed significantly lower glucose area under the curve (AUC) compared with $\mathrm{WB}, \mathrm{BT}$ and $\beta$ GBT $(\mathrm{p}<0.05)$. Insulin responses were similar between breads but significantly $(\mathrm{p}<0.05)$ different between time points (fasting vs $30 \mathrm{~min}, 30 \mathrm{~min}$ vs $120 \mathrm{~min}, 30 \mathrm{~min}$ vs $180 \mathrm{~min}$ ) (Fig. 2).

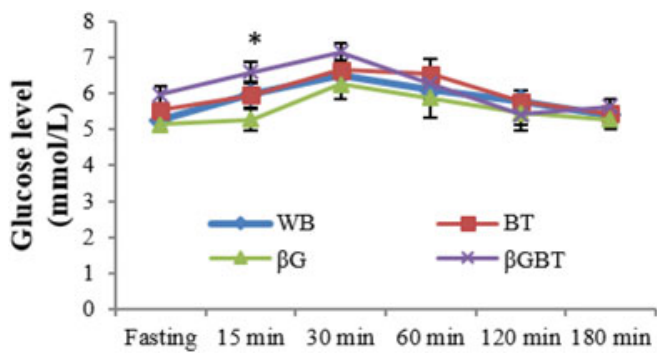

Fig. 1. Results are expressed as mean (SEM). Asterisk $(*)$ indicates significant $(\mathrm{p}<0.05)$ difference between bread.

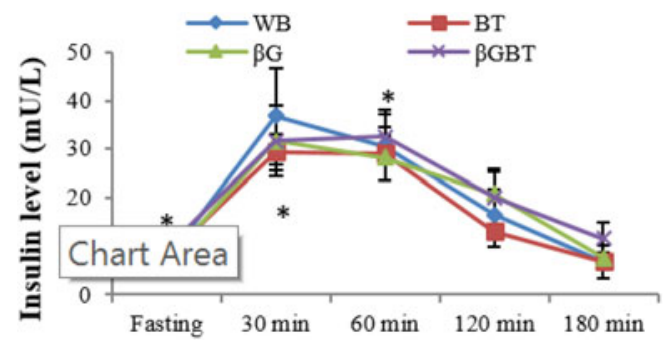

Fig. 2. Results are expressed as mean (SEM). Asterisk $(*)$ indicates significant $(\mathrm{p}<0.05)$ difference between bread.

This study suggests no beneficial effect of adding black tea in $\beta$-glucan bread on glucose and insulin responses. Future study is required to determine whether there are any significant dose and long-term effects of adding $\beta$-glucan and black tea to bread.

1. World Health Organization W. Facts about overweight and obesity. 2015 [cited 201516 December 2015]; Available from: http://www.who.int/mediacentre/factsheets/fs311/en/.

2. British Nutrition Foundation B. How much fibre do we need? 2016 [cited 20165 January 2016]; Available from: https://www.nutrition.org.uk/ healthyliving/basics/fibre.html.

3. Marlett JA, McBurney MI, Slavin JL. (2002). Position of the American Dietetic Association: health implications of dietary fiber. J Am Diet Assoc. 102(7), 993-1000.

4. Yahya HM, Day A, Lawton C, Myrissa K, Croden F, Dye L, et al. (2015). Dietary intake of 20 polyphenol subclasses in a cohort of UK women. Eur J Nutr.

5. Tresserra-Rimbau A, Rimm EB, Medina-Remon A, Martinez-Gonzalez MA, Lopez-Sabater MC, Covas MI, et al. (2014). Polyphenol intake and mortality risk: a re-analysis of the PREDIMED trial. BMC Med. 12, 77.

6. AM MJ, Edwards CA, Combet E, Ibrahim M, Garcia AL. (2015). Combined effects of added beta glucan and black tea in breads on starch functionality. Int J Food Sci Nutr. 66(2), 159-65. 